\title{
Topological Charge Fluctuations and Low-Lying Dirac Eigenmodes
}

\author{
S.J. Dong ${ }^{\mathrm{a}}$, T. Draper ${ }^{\mathrm{a}}$, I. Horváth ${ }^{\mathrm{a} *}$, N. Isgur ${ }^{\mathrm{b}}$, F.X. Lee ${ }^{\mathrm{bc}}$, J. McCune $^{\mathrm{d}}$, J.B. Zhang ${ }^{\mathrm{e}}$, H.B. Thacker ${ }^{\mathrm{d}}$ \\ a Department of Physics \& Astronomy, University of Kentucky, Lexington, KY 40506, USA \\ bJefferson Lab, 12000 Jefferson Avenue, Newport News, VA 23606, USA \\ ${ }^{c}$ Center for Nuclear Studies, George Washington University, Washington, DC 20052, USA \\ dDepartment of Physics, University of Virginia, Charlottesville, VA 22901, USA

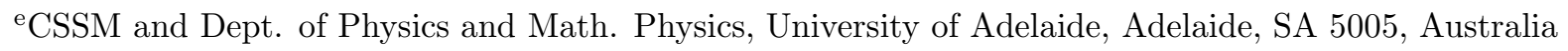

\begin{abstract}
We discuss the utility of low-lying Dirac eigenmodes for studying the nature of topological charge fluctuations in QCD. The implications of previous results using the local chirality histogram method are discussed, and the new results using the overlap Dirac operator in Wilson gauge backgrounds at lattice spacings ranging from $a \approx 0.04 \mathrm{fm}$ to $a \approx 0.12 \mathrm{fm}$ are reported. While the degree of local chirality does not change appreciably closer to the continuum limit, we find that the size and density of local structures responsible for chiral peaking do change significantly. The resulting values are in disagreement with the assumptions of the Instanton Liquid Model. We conclude that the fluctuations of topological charge in the QCD vacuum are not locally quantized.
\end{abstract}

The instanton (semiclassical) picture of the QCD vacuum has undergone several refinements since its beginnings as a dilute instanton gas, but there are two cornerstones that are inherent in this approach. First, it is imagined that the gauge field fluctuates in such a way as to form the lumps of approximately quantized topological charge. More precisely, it is assumed that there are regions of strong gauge fields that can be enclosed by a hypersurface on which the gauge field is approximately pure gauge. The topological charge contained in such regions thus have approximately quantized values, and we will refer to this situation as "lumpy vacuum" or vacuum with locally quantized topological charge. Secondly, the gauge field in such lumps is assumed to be overwhelmingly (anti)self-dual. Rather successful phenomenology, the Instanton Liquid Model (ILM) [1], has been built around these assumptions. The basic characteristics of the ILM are the average instanton radius $\rho \approx 1 / 3 \mathrm{fm}$ and density $n \approx 1 \mathrm{fm}^{-4}$ with values rather tightly restricted.

Using fermionic response as a signal for gauge

\footnotetext{
*The speaker at Lattice 2001.
}

fluctuations is a plausible strategy on the lattice, since lattice gauge fields are quite rough and believed to be affected strongly by artifacts at the scale of the lattice spacing. The attemps to eliminate the unphysical part of UV fluctuations directly from the gauge field are quite subjective and significantly biased as to the local structure of the vacuum they reveal. Indeed, cooling inevitably leads to lumpy and self-dual gauge fields if global topology is preserved in the process. Similarly, smoothing is biased towards the lumpy vacuum but not necessarily towards self-duality.

The eigenset of the Dirac operator fully encodes the information about the topological fluctuations of the underlying gauge field. While relying on the complete eigenset is impractical, the importance of these fluctuations mainly rests in their influence on the propagation of light quarks which, in turn, depends crucially on the low-lying modes. Hence, it is plausible to look for the imprints of topological charge fluctuations in Dirac near-zero modes [2]. This approach actually represents a form of smoothing because the infrared modes are least sensitive to the short-distance fea- 
tures atop larger structures in the gauge potential. However, "fermionic smoothing" is determined by the relevant fermionic dynamics, thus eliminating the subjective element.

While lattice fermion is not unique (different fermions smooth differently), all good actions should give consistent results sufficiently close to the continuum limit. However, to make meaningful comparisons, the same fermionic action should be used as the lattice spacing changes. There appears to be an interesting complementarity here of the type "degree of chiral symmetry $\times$ resolution = const". Valid ultralocal actions can not have exact chiral symmetry [3], but they can have perfect lattice "resolution" in the sense that the fermion only feels the gauge potential at its location. The actions with exact chiral symmetry, however, must be non-ultralocal and their resolution is poor. The advantage of the overlap operator over the Wilson-Dirac operator which still reflects the global topology quite well and has perfect resolution, is thus not obvious a priori.

A concrete proposal along these lines is the investigation of chiral orientation parameter $X(n)$ in the low eigenmodes [2]. $X(n)$ is a local angle in the $\left|\psi_{L}\right|-\left|\psi_{R}\right|$ plane, normalized to lie in the interval $[-1,1]$. This is quite useful because one can argue that the distribution of this quantity over active points on the lattice will have a predictable qualitative behaviour when the field is lumpy or self-dual. For a lumpy configuration, the emergence of topological near-zero-modes is expected, and the distribution of $X$ should be strongly peaked at the extremal values. When the gauge field exhibits extended regions of strong self-dual fields, the distribution should tend to be peaked as well. We emphasize that these two cases can happen independently, e.g. the lumpy field does not have to be self-dual for the distribution to be peaked. Consequently, such a qualitative behaviour is a necessary condition for instanton-like vacuum but by no means sufficient!

The first study of local chirality with Wilson fermions on Wilson gauge backgrounds at lattice spacing $a \approx 0.17 \mathrm{fm}$ found results inconsistent with the instanton-like vacuum [2]. Later works with Wilson fermions at smaller lattice spacing and also with the overlap revealed however, that a visible degree of double-peaked behaviour is present [4]. Our overlap calculation on Wilson gauge fields at $a \approx 0.123,0.042 \mathrm{fm}$ gave the following results

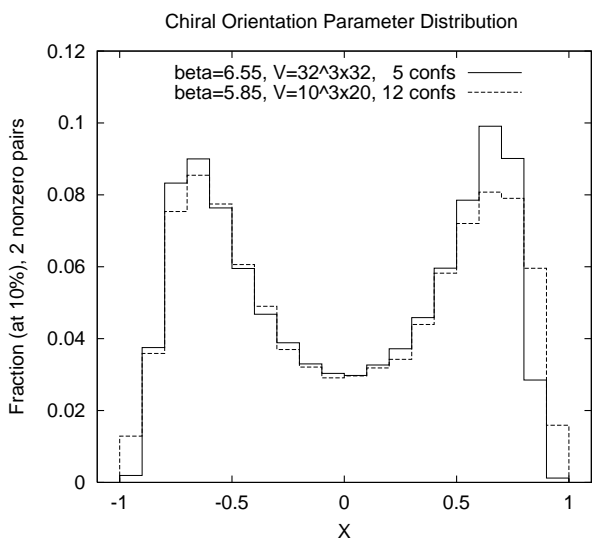

indicating that there does not appear to be a significant change in the degree of local chirality closer to the continuum limit. While the observed peaking does not imply the dominance of instantons, it does force us to look for quantitative comparisons rather than qualitative ones. It might be indeed quite revealing to compare the probability distribution of $X$ for an instanton liquid ensemble and for QCD using the overlap operator. To illustrate this point, we compare our results to the prediction of the dilute instanton gas model,

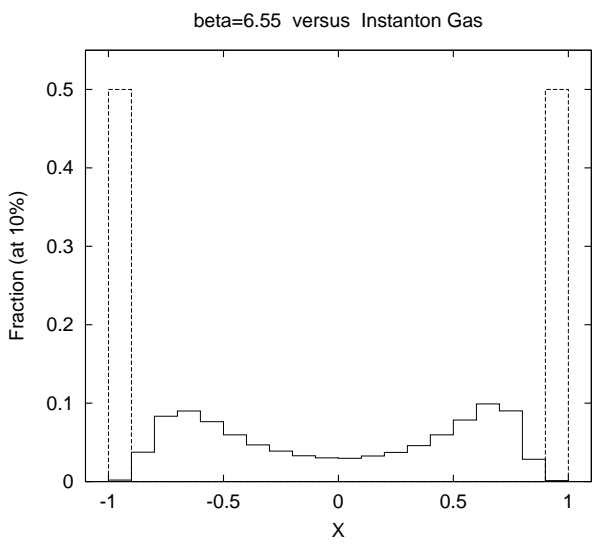

confirming that it is not applicable.

If the low-lying mode arises from mixing of t'Hooft "would be" zero modes associated with instantons, then the regions of coherent local chirality should be of a particular size and abun- 
dance. To check whether the behaviour of $X$ distribution can be ascribed to gauge structures with ILM parameters, we have found the local maxima over the distance $\sqrt{3}$ of density $\left(\psi^{+} \psi\right)$, and selected ones that (1) decay on average along the eight axis directions through distance $\sqrt{3}$ (this excludes accidental maxima and those not likely due to instantons), and (2) contribute to the peaks of $X$-distribution. We then determined the size of selected structures as (a) the radius of the largest hypersphere centered at the maximum and containing the sites with the same sign of chirality $\left(\psi^{+} \gamma_{5} \psi\right)$, and (b) the radius of the largest hypersphere within which density doesn't fall below $1 / 8$ of the center value (this corresponds to the radius in the case of an instanton). Using the first nonzero modes we found

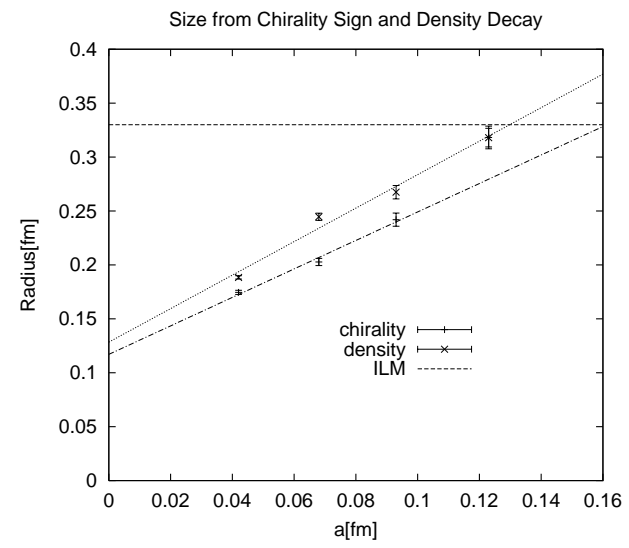

with values significantly below the ILM assumption close to continuum limit. Similarly, for the density of these structures we have obtained

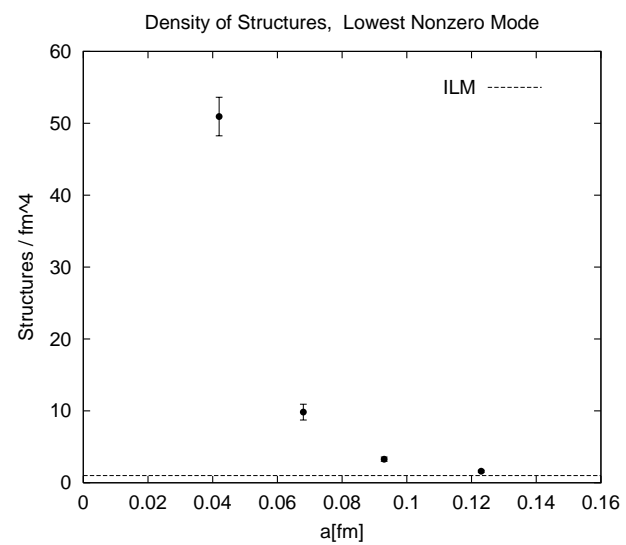

in a marked disagreement with the ILM. A few points need to be emphasized here. (A) There should not be any additional smoothing or other manipulation done on the low-lying eigenmodes. The lowest nonzero infrared mode provides a maximally smoothed imprint of topological charge fluctuations as seen by the propagating fermion. (B) The typical "size" of the chirality correlator in the lowest modes (representing an upper bound on the average size of underlying individual structures) at $a \approx 0.042 \mathrm{fm}$ is about $0.23 \mathrm{fm}$. (C) The possibility that the large density of structures we find is due to dislocations is unlikely and can be excluded in several ways.

How do we interpret our data? They are easy to understand if one abandons the assumption that topological charge in QCD vacuum is approximately locally quantized. For quantized topological charges, the calculable occurence of $0.15 \mathrm{fm}$ instantons should be negligible, but there is no such restriction for inhomogeneous fluctuations without quantization. Similarly, if the large number of structures we found corresponded to quantized "lumps", then the typical values of global topological charge (and susceptibility) would be much higher than we observe, but not necessarily so without quantization. We conclude that it is unlikely that the observed peaking of local chirality distribution is due to instanton-like objects with the parameters of the ILM. Instead, we find an evidence for serious breakdown of the semiclassical picture of the QCD vacuum [5].

\section{REFERENCES}

1. T. Schäfer, E. Shuryak, Rev. Mod. Phys. 70, 323 (1998).

2. I.Horváth, N.Isgur, J.McCune, H.B. Thacker, hep-lat/0102003.

3. I. Horváth, Phys. Rev. Lett. 81 (1998) 4063; I. Horváth, C.T. Balwe, R. Mendris, Nucl. Phys. B599, 283 (2001).

4. T.DeGrand, A.Hasenfratz, hep-lat/0103002 I.Hip et al, hep-lat/0105001; R.Edwards, U.Heller, hep-lat/0105004; T.Blum et al, hep-lat/0105006.

5. E. Witten, Nucl. Phys. B149, 285, (1979). 